\title{
Measuring Gait Speed in the Out-Patient Clinic: Methodology and Feasibility
}

\author{
Craig Karpman MD, Nathan K LeBrasseur PhD, Zachary S DePew MD, \\ Paul J Novotny, and Roberto P Benzo MD MSc
}

\begin{abstract}
BACKGROUND: Gait speed is a simple physical function measure associated with key outcomes in the elderly population. Gait speed measurements may improve clinical care in patients with COPD. However, there is a knowledge gap about the reliability and variability of gait speed testing protocols in COPD. We evaluated established techniques of measuring gait speed in patients with COPD and assessed feasibility of implementing gait speed as a routine vital sign in an out-patient clinic. METHODS: The usual 4-meter gait speed (4MGS) ("walk at a comfortable/natural pace"), maximal 4MGS ("walk as fast as you can safely"), usual 10-meter gait speed (10MGS), and maximal 10MGS of subjects with stable COPD were measured. Walks were measured using a stopwatch and automated timing system. For the feasibility/implementation phase, patients from the entire spectrum of respiratory diseases completed acceptability surveys, and clinical assistants administered gait speed measurements using an automated timing system. Time to train and to administer the test and acceptability by the staff were evaluated. RESULTS: Seventy subjects enrolled; $60 \%$ were men, and the mean age \pm SD was $69 \pm 10$ years. All methods showed excellent test-retest reliability (intraclass correlation coefficient of $0.95-0.97$ ). The difference between the two timing systems did not exceed the suggested minimal clinically important difference of $0.1 \mathrm{~m} / \mathrm{s}$ for the usual pace instructions but did exceed $0.1 \mathrm{~m} / \mathrm{s}$ for maximal pace walks. The difference between 4MGS and 10MGS was $0.13 \pm 0.10 \mathrm{~m} / \mathrm{s}$. FEASIBILITY: Most subjects reported that gait speed measurement prior to clinic appointment was very acceptable $(66 \%)$ or acceptable $(33 \%)$. Time added to clinic visit measuring 4MGS was $95 \pm 20$ seconds, and clinical assistants reported gait speed measurements as very acceptable $(60 \%)$, acceptable $(30 \%)$, and somewhat acceptable $(10 \%)$. CONCLUSIONS: Gait speed is a reliable measure in COPD, regardless of instructed pace, distance, or timing mechanism; however, adhering to one protocol is suggested. 4MGS was easily implemented into clinical practice with high acceptability by patients and clinic staff. Key words: gait; pulmonary disease; chronic obstructive; methods; feasibility studies; ambulatory care facilities; patient acceptance of health care. [Respir Care 2014;59(4):531-537. (O) 2014 Daedalus Enterprises]
\end{abstract}

\section{Introduction}

Short-distance gait speed is a reliable measure associated with falls, ${ }^{1}$ hospitalizations, ${ }^{2}$ disability,${ }^{3}$ and survival

Drs Karpman, DePew, and Benzo are affiliated with the Division of Pulmonary and Critical Care Medicine; Dr LeBrasseur is affiliated with the Division of Physical Medicine and Rehabilitation; and Dr Novotny is affiliated with the Department of Biomedical Statistics and Informatics, Mayo Clinic, Rochester, Minnesota.

This research has been presented as a poster presentation at the 2013 American Thoracic Society Meeting, held May 17-22, 2013, in Philadelphia, Pennsylvania. in older adults. ${ }^{4,5}$ Gait speed has been evaluated in numerous patient populations, including older individuals with neurologic, ${ }^{6,7}$ musculoskeletal, ${ }^{8,9}$ and cardiac disease. ${ }^{10}$ The routine measure of gait speed has been proposed in the

\footnotetext{
This work was supported by National Institutes of Health grant 1R01CA163293-01 from NHLBI (Primary investigator: RP Benzo). This publication was also made possible by Clinical and Translational Science Award (CTSA) grant UL1 TR000135 from the National Center for Advancing Translational Sciences (NCATS), a component of the National Institutes of Health (NIH). Its contents are solely the responsibility of the authors and do not necessarily represent the official view of NIH. The authors have disclosed no conflicts of interest.
} 


\section{Gait Speed Methodology and Feasibility}

elderly population to be implemented as a vital sign. ${ }^{11}$ Gait speed may be a clinically important measure in COPD. Recent studies have shown a link between gait speed and exercise capacity for patients with COPD. ${ }^{12,13}$ However, those studies did not evaluate the several commonly described gait speed protocols and have not established the effect of distance walked, pace, or timing methods on the reliability and reproducibility of gait speed results in patients with COPD. Prior studies using fast pace ${ }^{14,15}$ have not investigated the commonly used distances (4 and 10 meters) or the possible timing systems. Variability in the methodology is believed to affect clinical interpretation and implementation of the gait speed measures. ${ }^{16,17}$

In addition, the feasibility of implementing gait speed measurements into a clinical setting has not been thoroughly evaluated, and previous reports do not provide a comprehensive description of protocols or appraisal of realworld implementation. Our goals were, first, to evaluate the reliability and validity of several previously described protocols for measuring usual and maximal gait speed in COPD and, second, to assess the feasibility of implementing a gait speed measure as a vital sign in a respiratory out-patient clinic.

\section{Methods}

\section{Subjects}

Participants with clinically stable COPD were prospectively recruited from an out-patient pulmonary clinic. Inclusion criteria consisted of: (1) diagnosis of COPD based on the Global Initiative for Chronic Obstructive Lung Disease 2011 guidelines, ${ }^{18}$ (2) stable respiratory condition one month prior to the study, and (3) ability to walk without limitation by a predominant orthopedic or neurologic disease ("when walking, are you more limited by breathlessness or by pain, unsteadiness, or weakness?"). Clinical characteristics collected included age, sex, body mass index (BMI), and subjective dyspnea as measured by the Modified Medical Research Council (MMRC) dyspnea scale. ${ }^{19}$ Approval was obtained from the Mayo Foundation Institutional Review Board.

\section{Gait Speed Measurements}

Gait speed measurements were performed in a flat and unobstructed clinic hallway. All measurements were ob-

\footnotetext{
Correspondence: Roberto P Benzo MD MSc, Mindful Breathing Laboratory, Division of Pulmonary and Critical Care Medicine, Mayo Clinic, 200 1st Street SW, Rochester, MN 55905. E-mail: benzo.roberto@mayo. edu.
}

DOI: $10.4187 /$ respcare. 02688

\section{QUICK LOOK}

\section{Current knowledge}

Gait speed is simple physical function measure associated with key outcomes in the elderly population. However, there is a knowledge gap about the reliability and variability of gait speed testing in patients with COPD.

\section{What this paper contributes to our knowledge}

Gait speed is a reliable measure in patients with COPD regardless of instructed pace, distance, or timing mechanism. The 4-meter gait speed protocol was easily implemented into clinical practice with high acceptability by patients and staff.

tained by one trained investigator $(\mathrm{CK})$, and each of the gait speed protocols was performed two times for each subject with a 5-10-second break between trials. Each walk was performed with a 2 -meter rolling start, where the participant is already walking upon entering the measuring area (Fig. 1). Canes, walkers, and supplemental oxygen were used if the subject normally used the equipment in daily activity. Usual gait speed over a 4-meter course (4MGS), maximal 4MGS, usual gait speed over a 10meter course (10MGS), and maximal 10MGS were measured.

Usual 4-Meter Gait Speed. Two cones were placed 8 meters apart, and an automated timing system was set up 2 meters after the first cone and 2 meters before the second cone, as shown in Figure 1. We used a dual-beam wireless infrared timing system (TracTronix, Lenexa, Kansas) on 6-inch tripods with a preset 2-second delay (allowing for a rolling oxygen tank, walkers, or canes to be used). This provided a 2-meter acceleration zone, a 4-meter timing area, and a 2-meter deceleration zone. Subjects were instructed to "walk at a comfortable/normal pace" from one cone to the other. The time to walk 4 meters was measured using the automated timing system simultaneously with a manual stopwatch. The automated timing system would activate once the first timer plane was broken by the participant and would stop once the second timer plane was broken. For the manual measure, the test administrator stood in the middle of the 4-meter course and started the stopwatch when the participant's first foot completely crossed into the timing area and stopped the stopwatch once the participant's first foot completely came out of the timing area.

Maximal 4-Meter Gait Speed. Maximal 4MGS was measured in the same fashion as usual 4MGS other than the walking instructions. Subjects were instructed to "walk as 


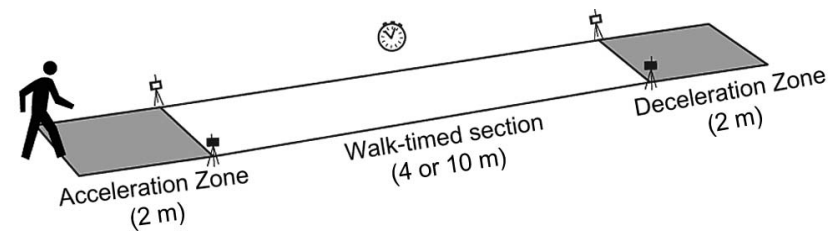

Fig. 1. Gait speed measurement.

fast as you can safely, without running" from one cone to the other. Maximal 4MGS was measured using both the automated timing system and the stopwatch.

Usual 10-Meter Gait Speed. Two cones were placed 14 meters apart, and an automated timing system was set up 2 meters after the first cone and 2 meters before the second cone. This provided a 2-meter acceleration zone, a 10meter timing area, and a 2-meter deceleration zone. Subjects were instructed to "walk at a comfortable/normal pace" from one cone to the other. Only the automated timing system was used for 10MGS measurements.

Maximal 10-Meter Gait Speed. Subjects were instructed to "walk as fast as you can safely" from one cone to the other. Only the automated timing system was used for maximal 10MGS measurements.

\section{Feasibility of Measuring Gait Speed in the Clinic}

Patient Acceptability. A separate convenience sample, not recruited for the methodology portion of the study, receiving out-patient care in the Pulmonary Department of the Mayo Clinic, Rochester, Minnesota, was recruited for the feasibility portion of the study. After subjects were checked in for their prescheduled appointment and had the standard vital signs checked by clinical assistants (heart rate, blood pressure, etc), the subjects were approached by study personnel (CK) and asked to perform usual and maximal 4MGS prior to the physician visit. After completion of the gait speed measurements, subjects were surveyed about the acceptability of the study procedures by using a 4-point Likert scale (not acceptable, somewhat acceptable, acceptable, and very acceptable). Demographics of the study participants were also collected.

Clinical Implementation. The second phase of the feasibility study involved training clinical assistants to set up the automated timing system and perform the gait speed measure as a routine clinical vital sign (Fig. 2). The time to train staff, the time to set up equipment, and the added time in clinic to administer the test were evaluated. Staff acceptability was assessed using an anonymous questionnaire with a 4-point Likert scale (not acceptable, somewhat acceptable, acceptable, and very acceptable).

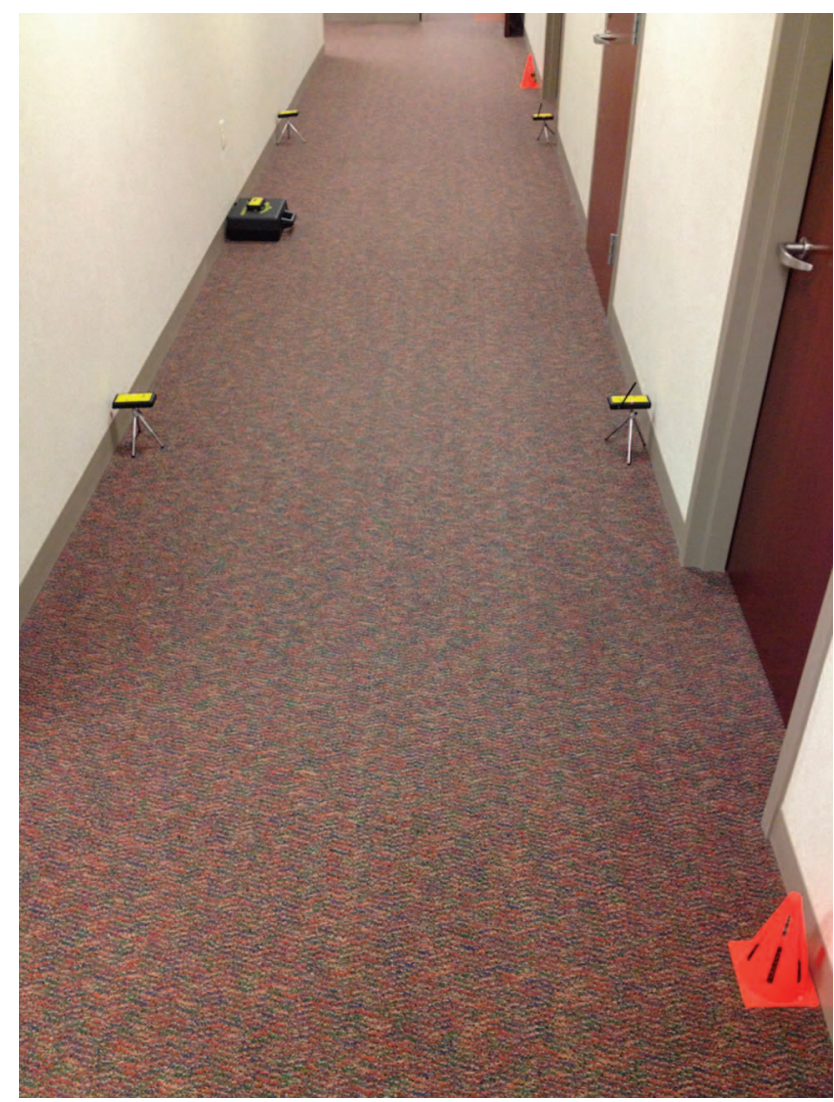

Fig. 2. Automated gate speed timing setup.

\section{Statistical Analysis}

Data were summarized as means $\pm \mathrm{SD}$. Intraclass correlation coefficient (ICC) calculations were used to evaluate the reliability between testing and retesting of 4MGS and 10MGS and between automated and manual timing for usual and maximal 4 MGS. ${ }^{20}$ Variability between repeat measurements was analyzed using standard error of measurement (SEM) and standard errors of measurements (SEM\%). The SEM provides the error value in the same unit as the initial measure, and the SEM\% describes the error in percent, allowing for comparison between tests that have different units. The validity of stopwatch measurements compared with an automated timer was evaluated using the Bland-Altman method and calculating the 95\% limits of agreement between the two timing systems. Since all gait speed measurements were performed twice, the fastest speed was used during analysis. ${ }^{3}$ Spearman's rank correlation was used for evaluation of the association between the timing systems throughout the spectrum of the gait speeds. For all analyses, $P \leq .05$ was considered statistically significant. 
Table 1. Walking Speeds

\begin{tabular}{lcc}
\hline \hline \multicolumn{1}{c}{ Walking Speed Protocol } & $\begin{array}{c}\text { Mean } \\
\text { Speed } \pm \text { SD } \\
(\mathrm{m} / \mathrm{s})\end{array}$ & $\begin{array}{c}\text { Minimum-Maximum } \\
(\mathrm{m} / \mathrm{s})\end{array}$ \\
\hline Usual 4MGS (stopwatch) & $1.13 \pm 0.23$ & $0.68-1.74$ \\
Usual 4MGS (timer) & $1.14 \pm 0.24$ & $0.68-1.82$ \\
Usual 10MGS (timer) & $1.27 \pm 0.24$ & $0.87-2.00$ \\
Maximal 4MGS (stopwatch) & $1.68 \pm 0.37$ & $0.98-2.50$ \\
Maximal 4MGS (timer) & $1.69 \pm 0.38$ & $0.93-2.67$ \\
Maximal 10MGS (timer) & $1.77 \pm 0.39$ & $1.05-2.78$ \\
& & \\
& & \\
Data are presented as mean \pm SD. & & \\
4MGS = 4-meter gait speed & & \\
10MGS = 10-meter gait speed & & \\
\hline
\end{tabular}

\section{Results}

Seventy subjects enrolled in the methodology study, and each of the four gait speed measures was performed. The mean age \pm SD of the subjects was $66 \pm 9$ years, with a BMI of $30 \pm 6 \mathrm{~kg} / \mathrm{m}^{2} ; 43$ (60\%) were male, and 69 (99\%) were white. The severity of COPD was moderate to severe with a percent predicted $\mathrm{FEV}_{1}\left(\mathrm{FEV}_{1} \%\right)$ of $53 \pm 18$, and 11 subjects $(16 \%)$ were on supplemental oxygen. Subjects were short of breath with minimal activity based on an MMRC score of $2 \pm 1$. The mean gait speeds are summarized in Table 1.

\section{Test-Retest Reliability}

The 4MGS measurements showed excellent test-retest reliability at both usual and maximal pace and when timed with either the automated timing system or a manual stopwatch (all ICCs $\geq 0.95$; detailed in Table 2). The usual and maximal 10MGS measurements also demonstrated exceptional agreement for test-retest with ICCs of 0.97 and narrow confidence intervals. We found a low measure of variability for $4 \mathrm{MGS}$ and 10MGS measures with SEM values ranging from 0.04 to $0.08 \mathrm{~m} / \mathrm{s}$ (Table 2).

\section{Timing Method Validity}

First, we evaluated the correlation between the stopwatch and automated timing systems using ICC, and we found excellent values of $0.99(0.98-0.99)$ for usual 4MGS and $0.99(0.98-0.99)$ for maximal 4MGS. We then used Bland-Altman analysis to plot the difference between the two methods and found a mean difference of $0.01 \mathrm{~m} / \mathrm{s}$ for both usual and maximal pace. The $95 \%$ limit of agreement for usual pace was -0.10 to $0.08 \mathrm{~m} / \mathrm{s}$ and was larger at -0.15 to $0.12 \mathrm{~m} / \mathrm{s}$ for maximal pace (Fig. 3). As evident by the Bland-Altman figures, the variation between the two timing systems did not exceed $0.1 \mathrm{~m} / \mathrm{s}$ for the usual pace instructions, and the best agreement was seen at the slower gait speeds. The maximal 4MGS Bland-Altman figure shows that the variation increased with faster speeds and the $95 \%$ limit of agreement exceeded $0.1 \mathrm{~m} / \mathrm{s}$.

\section{MGS Versus 10MGS Measurements}

Usual 4MGS and usual 10MGS had a difference of $0.12 \pm 0.10 \mathrm{~m} / \mathrm{s}(P<.001)$ and the difference between maximal $4 \mathrm{MGS}$ and $10 \mathrm{MGS}$ was also significant at $0.06 \pm 0.14 \mathrm{~m} / \mathrm{s}(P<.001)$. Bland-Altman analysis determined a mean difference of $0.12 \mathrm{~m} / \mathrm{s}$ and a $95 \%$ limit of agreement of -0.07 to $0.31 \mathrm{~m} / \mathrm{s}$ for usual pace and a mean difference of $0.08 \mathrm{~m} / \mathrm{s}$ and a $95 \%$ limit of agreement of -0.20 to $0.36 \mathrm{~m} / \mathrm{s}$ for maximal pace (Fig. 4).

\section{Patient Acceptability}

A separate cohort of 100 subjects participated in the acceptability portion of the feasibility study. The age was $63 \pm 15$ years, and the most common disease processes were COPD, interstitial lung disease, and asthma. The average usual 4MGS as measured by the automated timing system was $1.1 \pm 0.26 \mathrm{~m} / \mathrm{s}$, and maximal $4 \mathrm{MGS}$ was $1.59 \pm 0.42 \mathrm{~m} / \mathrm{s}$. Ninety-nine of 100 subjects reported that the gait speed measurement prior to the clinic appointment was either very acceptable (66\%) or acceptable (33\%). One subject reported that the 4MGS measurement was somewhat acceptable.

\section{Clinical Implementation}

Gait speed was implemented as a vital sign in the outpatient clinic for a 60-day period. During this time period, every morning prior to clinic, the cones and automated timers were set up by clinical assistants in a low traffic hallway to conduct 4 MGS measurements. The time to set up the equipment each day was $3 \pm 1 \mathrm{~min}$. To evaluate the time added to the standard clinic intake procedure, we measured the time it took to complete the 4MGS measurement for 10 randomly selected subjects. The average time added to the clinic visit by measuring 4MGS was $95 \pm 20$ seconds. Using the automated timers, 9 of 10 clinical assistants reported that collecting the gait speed measurements during clinic as very acceptable ${ }^{6}$ or acceptable. ${ }^{3}$ One clinical assistant reported the measurement as somewhat acceptable. The time to train staff ranged from 9 to $12 \mathrm{~min}$.

\section{Discussion}

Gait speed measurements are reliable in patients with COPD regardless of instructed pace (usual or maximal), 
Table 2. Test-Retest Reliability of Various Gait Speed Measurements

\begin{tabular}{lccc}
\hline \hline \multicolumn{1}{c}{ Test } & ICC (95\% CI) & Mean Difference (95\% CI) & SEM (m/s) \\
\hline Usual 4MGS (stopwatch) & $0.95(0.92-0.97)$ & $0.01(-0.03$ to 0.01) & 0.05 \\
Usual 4MGS (timer) & $0.95(0.91-0.97)$ & $<0.01(-0.02$ to 0.02) & 0.05 \\
Usual 10MGS (timer) & $0.97(0.95-0.98)$ & $<0.01(-0.02$ to 0.01$)$ & 0.04 \\
Maximal 4MGS (stopwatch) & $0.95(0.93-0.97)$ & $0.02(-0.04$ to 0.01$)$ & 0.08 \\
Maximal 4MGS (timer) & $0.95(0.92-0.97)$ & $0.02(-0.05$ to 0.01$)$ & 0.08 \\
Maximal 10MGS (timer) & $0.97(0.96-0.98)$ & $0.03(-0.05$ to $<0.01)$ & 0.07 \\
& & & 4.8 \\
4MGS = 4-meter gait speed & & & 4.0 \\
10MGS = 10-meter gait speed & & & \\
SEM = standard error of measurement & & & \\
SEM\% = standard errors of measurements & & & \\
ICC = intraclass correlation coefficient & & & \\
\hline
\end{tabular}
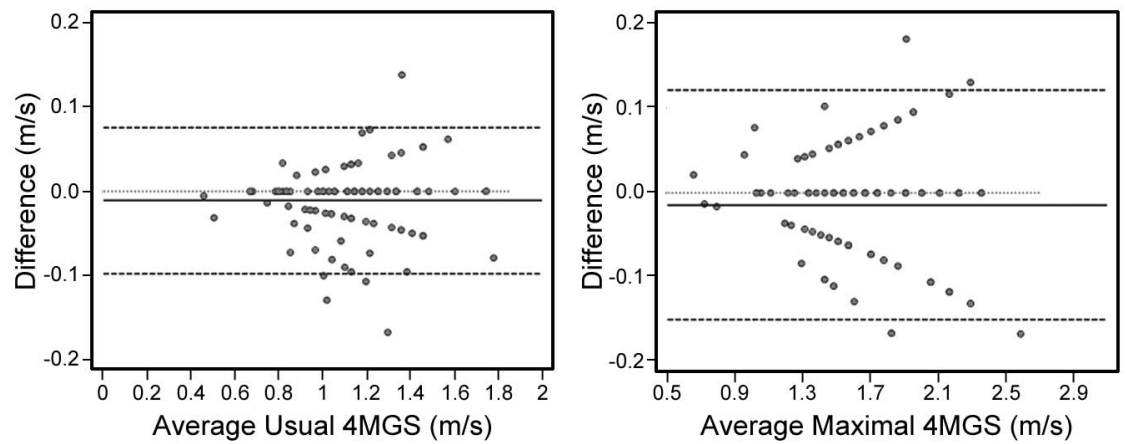

Fig. 3. Difference between stopwatch and timer for usual and maximal 4-meter gait speed (4MGS).
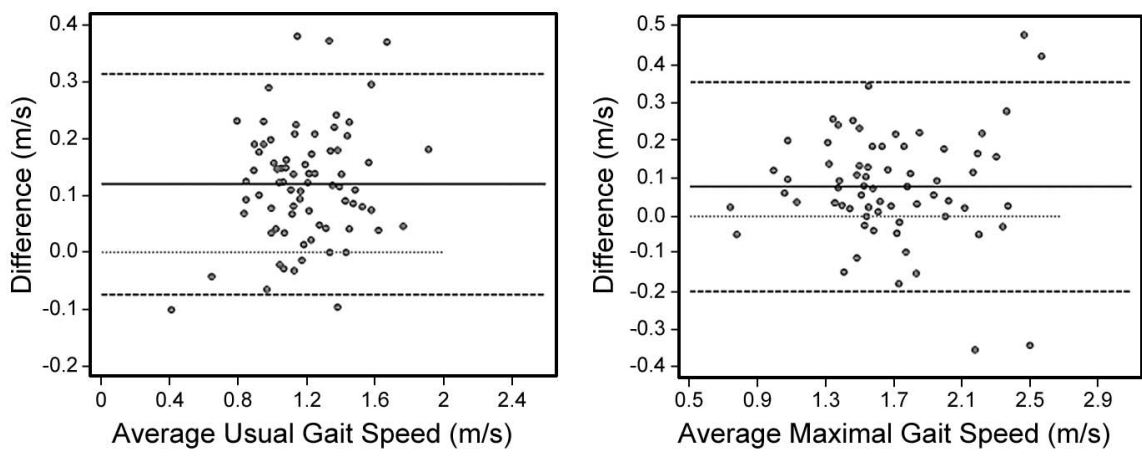

Fig. 4. Difference between usual and maximal 4-meter gait speed vs 10-meter gait speeds.

distance (4 or 10 meters), or timing system (stopwatch or automated timer). Our feasibility study showed that gait speed was easily incorporated into a clinical setting with high acceptance by patients and staff with the use of an automated timing system.

Our work adds to the field by comprehensively evaluating the numerous technical variables involved in measuring gait speed in COPD and using the more commonly described distances of 4 and 10 meters. The excellent reliability, regardless of distance or timing mechanism, seen in our study is consistent with prior work in this field. The ICC values for test-retest and for agreement between tim- ing systems also ranged from 0.96 to 1.00 in the study by Peters et $\mathrm{al}^{21}$ when evaluating healthy older adults. Our group had better test-retest reliability for the usual 10meter gait speed (ICC of 0.97 vs 0.87 ) when comparing with the usual 30-meter gait speed measured in a smaller COPD cohort. ${ }^{14}$

This study showed that in patients with COPD, there is good agreement between stopwatch and automated timing system measurements for usual gait speed measurements. That agreement did not hold true with maximal walking speeds. The 95\% CI for agreement between timing systems for maximal 4MGS reached the $0.1 \mathrm{~m} / \mathrm{s}$ cutoff. The 


\section{Gait Speed Methodology and Feasibility}

latter may or may not be clinically important since the minimum clinically important difference for gait speed of $0.1 \mathrm{~m} / \mathrm{s}$ has been postulated for usual but not for maximal gait speed. ${ }^{11}$ If maximal gait speed is used, based on this work, stopwatch and automated timing systems cannot be used interchangeably. We presume that the automated timing system would be more accurate than the handheld stopwatch for maximal pace short-distance walks, which take only a few seconds to complete. Our finding of minimal variability for the timing systems with the usual pace gait speed measurements is consistent with prior studies. ${ }^{21}$

We also found that there was a significant difference in gait speeds measured between longer (10 meter) and shorter (4 meter) walks, and that difference was $>0.1 \mathrm{~m} / \mathrm{s}$ (meaningful difference), suggesting that is better to stick to one protocol of gait speed measurement. Similar to our results, Peters et $\mathrm{al}^{21}$ identified enough variability between 4MGS and 10MGS that they would not use the measurements interchangeably. A large review also found that longer walks were, on average, faster than shorter walks in healthy older patients. ${ }^{17}$ The opposite was seen in the neurologic disease cohort from the same review, ${ }^{17}$ where the shorter walks were faster. There are also several prior studies showing no significant variation in gait speeds between shorter and longer walking distances. ${ }^{22}$ A plausible explanation for the difference in our study is an inadequate acceleration zone of 2 meters, and during a longer walk (10 meters), this limitation is masked by the extended duration of the test. Whether gait speed is actually faster in COPD when measured over a longer distance is unclear, but there is enough variability throughout studies that we would recommend consistently using a single distance.

\section{Feasibility}

With improved understanding of various gait speed protocols, we can better select the methods for implementation of gait speed assessment in the clinical setting. Using the information gleaned from the methodology section of this study, we set up a real-world feasibility study of measuring gait speed in an out-patient clinic. We found nearly unanimously positive results from subjects undergoing their clinic evaluations. We also evaluated the time to train and the overall burden for clinical assistants in administering the test, and we found positive results in both domains. We believe that this positive response is based on the simplicity and ease of performing the measures. Studenski et al. ${ }^{23}$ also evaluated the feasibility of measuring gait speed in the clinic of a cohort of veterans. They found that participants and health care professionals were accepting of the testing.

We chose to use the automated laser timing system for the feasibility portion of the study even when most studies measuring walking speed use a stopwatch as the timing mechanism. We wanted to use a method that required minimal training that could be completed in $<15 \mathrm{~min}$. In a tertiary clinical setting where numerous personnel are measuring the walking speed, a method that is simplest to train and with the lowest chance of variability between operators would be most effective in facilitating clinical implementation. As described in the Short Physical Performance Battery protocol provided by the National Institute on Aging (www.grc.nia.nih.gov, Accessed May 20, 2013), the stopwatch timing method requires operators to be trained with extensive and detailed instruction. However, using a stopwatch with instruction from the National Institute on Aging could also be a reasonable option for clinical use if the training time is available or if the cost of the automated laser timing system $(\$ 289$ in the United States) is a barrier. On the basis of our results, we would not recommend alternating between a stopwatch and a timing system if a maximal speed protocol is used.

\section{Limitations}

Although our objective was to comprehensively measure the possible gait speed protocols, we did not measure static start. Since this research was performed by recruiting from a busy clinic and patient schedule, there was a limitation of how many protocols could be tested at the same time. In addition, rolling start is thought to provide less variability in the results of gait speed by not having the acceleration phase ${ }^{17}$ of the static start gait speed measure, which can increase the noise-to-signal ratio. Future studies may be warranted to evaluate static and rolling start in the same cohort. The feasibility portion of our study included a heterogeneous group of respiratory diseases, but we used a gait speed protocol developed in a cohort of patients with COPD. We also did not compare the feasibility of automated timer versus stopwatch, and the acceptability was not compared with 10MGS measurements.

\section{Conclusions}

Gait speed is a reliable measure in COPD regardless of instructed pace, distance, or timing mechanism. On the basis of this work, we recommend using one method consistently: same distance and speed instructions all of the time. Four-meter gait speed was easily implemented into clinical practice with excellent acceptability by patients and clinic staff using an automated timing system. We hope that these results inform and ignite health care providers to incorporate this feasible and useful measure into daily clinical practice.

\section{ACKNOWLEDGMENTS}

We thank Marnie Wetzstein, RN and Johanna Hoult from the Pulmonary Clinical Research Unit, the Mayo Clinic Pulmonary Function Lab, and 


\section{Gait Speed Methodology and Feasibility}

Mayo Clinic Pulmonary clinical assistants for their assistance with logistical management and recruitment for the study. We thank Molly E. McMahon, Mayo Clinic Center for Innovation, for creating the gait speed illustration in this manuscript.

\section{REFERENCES}

1. Biderman A, Cwikel J, Fried AV, Galinsky D. Depression and falls among community dwelling elderly people: a search for common risk factors. J Epidemiol Community Health 2002;56(8):631-636.

2. Montero-Odasso M, Schapira M, Soriano ER, Varela M, Kaplan R, Camera LA, et al. Gait velocity as a single predictor of adverse events in healthy seniors aged 75 years and older. J Gerontol A Biol Sci Med Sci 2005;60(10):1304-1309.

3. Cesari M, Kritchevsky SB, Penninx BWHJ, Nicklas BJ, Simonsick EM, Newman AB, et al. Prognostic value of usual gait speed in well-functioning older people-results from the Health, Aging and Body Composition Study. J Am Geriatr Soc 2005;53(10):1675-1680.

4. Studenski S, Perera S, Patel K, Rosano C, Faulkner K, Inzitari M, et al. Gait speed and survival in older adults. JAMA 2011;305(1):5058.

5. Hardy SE, Perera S, Roumani YF, Chandler JM, Studenski SA. Improvement in usual gait speed predicts better survival in older adults. J Am Geriatr Soc 2007;55(11):1727-1734.

6. Dodge HH, Mattek NC, Austin D, Hayes TL, Kaye JA. In-home walking speeds and variability trajectories associated with mild cognitive impairment. Neurology 2012;78(24):1946-1952.

7. Ries JD, Echternach JL, Nof L, Gagnon Blodgett M. Test-retest reliability and minimal detectable change scores for the timed "up \& go" test, the six-minute walk test, and gait speed in people with Alzheimer disease. Phys Ther 2009;89(6):569-579.

8. Kaufman KR, Hughes C, Morrey BF, Morrey M, An K-N. Gait characteristics of patients with knee osteoarthritis. J Biomech 2001; 34(7):907-915.

9. Ostchega Y, Dillon CF, Lindle R, Carroll M, Hurley BF. Isokinetic leg muscle strength in older Americans and its relationship to a standardized walk test: data from the national health and nutrition examination survey 1999-2000. J Am Geriatr Soc 2004;52(6):977982.

10. Afilalo J, Eisenberg MJ, Morin JF, Bergman H, Monette J, Noiseux $\mathrm{N}$, et al. Gait speed as an incremental predictor of mortality and major morbidity in elderly patients undergoing cardiac surgery. J Am Coll Cardiol 2010;56(20):1668-1676.
11. Fritz S, Lusardi M. White paper: "walking speed: the sixth vital sign." J Geriatr Phys Ther 2009;32(2):46-49.

12. Kon SS, Patel MS, Canavan JL, Clark AL, Jones SE, Nolan CM, et al. Reliability and validity of the 4-metre gait speed in COPD. Eur Respir J 2013;42(2):333-340.

13. DePew Z, Karpman C, Novotny P, Benzo R. Correlations between gait speed, six-minute walk, physical activity, and self-efficacy in severe chronic lung disease. Respir Care 2013 [Epub ahead of print] doi:10.4187/respcare.02471

14. Andersson M, Moberg L, Svantesson U, Sundbom A, Johansson H, Emtner M. Measuring walking speed in COPD: test-retest reliability of the 30-metre walk test and comparison with the 6-minute walk test. Prim Care Respir J 2011;20(4):434-440.

15. Bohannon RW. Comfortable and maximum walking speed of adults aged 20-79 years: reference values and determinants. Age Ageing 1997;26(1):15-19.

16. Graham JE, Ostir GV, Fisher SR, Ottenbacher KJ. Assessing walking speed in clinical research: a systematic review. J Eval Clin Pract 2008;14(4):552-562.

17. Graham JE, Ostir GV, Kuo YF, Fisher SR, Ottenbacher KJ. Relationship between test methodology and mean velocity in timed walk tests: a review. Arch Phys Med Rehabil 2008;89(5):865-872.

18. Vestbo J, Hurd SS, Rodriguez-Roisin R. The 2011 revision of the global strategy for the diagnosis, management and prevention of COPD (GOLD)-why and what? Clin Respir J 2012;6(4):208-214.

19. Hajiro T, Nishimura K, Tsukino M, Ikeda A, Koyama H, Izumi T. Analysis of clinical methods used to evaluate dyspnea in patients with chronic obstructive pulmonary disease. Am J Respir Crit Care Med 1998;158(4):1185-1189.

20. Lin LI. A concordance correlation coefficient to evaluate reproducibility. Biometrics 1989;45(1):255-268.

21. Peters DM, Fritz SL, Krotish DE. Assessing the reliability and validity of a shorter walk test compared with the 10-meter walk test for measurements of gait speed in healthy, older adults. J Geriatr Phys Ther 2013;36(1):24-30.

22. Peel NM, Kuys SS, Klein K. Gait speed as a measure in geriatric assessment in clinical settings: a systematic review. J Gerontol A Biol Sci Med Sci 2013;68(1):39-46.

23. Studenski S, Perera S, Wallace D, Chandler JM, Duncan PW, Rooney E, et al. Physical performance measures in the clinical setting. J Am Geriatr Soc 2003;51(3):314-322. 